\title{
A 12-week randomized, double-blind, placebo-controlled multicenter study of choline-stabilized orthosilicic acid in patients with symptomatic knee osteoarthritis
}

Piet Geusens ${ }^{1,2,3^{*}}$, Karel Pavelka ${ }^{4}$, Jozef Rovensky ${ }^{5}$, Johan Vanhoof ${ }^{3}$, Nathalie Demeester ${ }^{6}$, Mario Calomme ${ }^{6}$ (D) and Dirk Vanden Berghe ${ }^{7}$

\begin{abstract}
Background: The aim of this study was to assess the efficacy of choline-stabilized orthosilicic acid (ch-OSA) in patients with symptomatic knee osteoarthritis (OA).

Methods: In a multicenter, double-blind, placebo-controlled study, 211 patients with knee OA (Kellgren and Lawrence grade II or III) and moderate to moderately severe pain were randomly allocated to ch-OSA or placebo for 12 weeks. The primary outcome was the change in the WOMAC pain subscale from baseline to week 12. Secondary outcomes were changes from baseline to week 12 in WOMAC total, WOMAC stiffness, WOMAC physical function, Subject Global Assessment and levels of cartilage degradation biomarkers C-terminal telopeptide of collagen type II (CTX-II) and cartilage oligomeric matrix protein (COMP). Pre-specified subgroup analyses included the effect of gender.

Results: A total of 166 (120 women, 46 men) patients were included in the analysis (87 and 79 in the ch-OSA and placebo group, respectively). In the total study population, no differences were observed between the two treatment groups for the different outcomes but significant treatment $x$ gender interactions were found. In men taking ch-OSA, a significant improvement in WOMAC total, WOMAC stiffness and WOMAC physical function as well as a lower increase in biomarker levels of cartilage degradation was observed, but not in women. The change in WOMAC pain showed a similar positive trend in men taking ch-OSA.
\end{abstract}

Conclusion: After 12 weeks of treatment, no effect was found of ch-OSA in the total study population on clinical parameters and biomarkers, but a gender interaction was observed. In men, ch-OSA was found effective in reducing symptoms of knee OA, which was associated with a slight but significant reduction of biomarkers that are related to cartilage degradation.

Trial registration: The study was registered retrospectively: ISRCTN88583133. Registration date: 2015-10-07.

Keywords: Osteoarthritis, Knee, Choline-stabilized orthosilicic acid, WOMAC, Cartilage degradation marker, Silicon, Choline

\footnotetext{
* Correspondence: drpgeusens@gmail.com

${ }^{1}$ Biomedical Research Institute (BIOMED), Hasselt University, Diepenbeek,

Belgium

${ }^{2}$ Rheumatology, Maastricht UMC, Maastricht, The Netherlands

Full list of author information is available at the end of the article
} 


\section{Background}

Osteoarthritis (OA) is one of the leading causes of functional disability and compromised quality of life in the elderly worldwide [1]. It is a chronic and slowly progressive disease, with the knee being the most affected weightbearing joint $[2,3]$. OA is a complex disease of joints, characterised by degradation of articular cartilage, changes of the subchondral bone and inflammation, leading to pain and stiffness in the joint $[4,5]$. At present, there are no safe OA therapeutic strategies approved that result in a concurrent structural modification and symptom improvement $[6,7]$. The treatment of OA is therefore, besides exercise and physiotherapy, still mainly based on analgesics, nonsteroidal anti-inflammatory drugs (NSAIDs) and surgical procedures [8].

Several risk factors for the development of knee OA have been identified, including age, obesity, injury and genetic profiles. Sex differences in knee OA incidence and prevalence have been reported as well, with females generally at higher risk [7, 9]. Moreover, the meta-analysis performed by Srikanth et al. demonstrated that women tend to have more severe knee OA, particularly after menopausal age [9].

Given the limitations of plain radiography and the high charges for magnetic resonance imaging (MRI), biochemical markers have gained considerable interest over the past 15 years $[10,11]$. However, in contrast to the wellestablished biochemical markers for bone diseases, the value of biomarkers in OA still needs further elucidation [12]. To this respect, Bauer et al. proposed the BIPED burden of disease, investigative, prognostic, efficacy of intervention and diagnostic - categories, providing a classification system for biochemical markers [13]. Among numerous candidates, $\mathrm{C}$-terminal telopeptide of collagen type II (CTX-II) and cartilage oligomeric matrix protein (COMP), which are two markers of cartilage degradation, have been reported to be the best performing biochemical markers across all BIPED categories [11].

Choline-stabilized orthosilicic acid (ch-OSA) is a specific complex of orthosilicic acid and choline that has been previously demonstrated to stimulate collagen synthesis which positively affects bone turnover [14] as well as surface and mechanical properties of the skin [15]. The choline component of ch-OSA was suggested to contribute to this effect [14], as it lowers plasma homocysteine levels [16]. More specifically, hyperhomocysteinemia has been shown to interfere with collagen cross-linking, leading to connective tissue pathology [17]. To this respect, it may be hypothesized that ch-OSA acts on both cartilage and subchondral bone, consequently rendering it a candidate for the treatment of OA. The aim of this study was to evaluate the symptomatic effects of the oral intake of chOSA on knee OA over a 12-week period. Additionally, CTX-II and COMP were analysed to assess the influence of ch-OSA on cartilage degradation.

\section{Methods}

A 12-week, international, multicenter, double-blind, randomized, parallel-group, placebo-controlled single-joint study in outpatients with symptomatic knee OA was performed. Participants were recruited either directly from the investigation centres (situated in Belgium (ReumaClinic, Genk and Diagnosecentrum, Lommel; both private hospitals in co-operation with Biomedical Research Institute, Hasselt University, Hasselt, Belgium and Rheumatology, Maastricht UMC, Maastricht, The Netherlands), Slovakia (National Institute of Rheumatic Diseases, Piestany; public hospital) and Czech Republic (Institute of Rheumatology, Prague; public hospital) or following pre-screening by general practitioners (Belgium).

\section{Patients}

Potential participants were initially assessed during a screening visit. The inclusion criteria defined eligible patients as men and women between 50 and 75 years of age with a diagnosis of primary knee OA according to the American College of Rheumatology criteria [18] for at least 12 weeks prior to randomization i.e. knee pain plus at least three of the following characteristics: $>50$ years, $<30$ min of morning stiffness, crepitus on active motion, bony tenderness, bony enlargement, no palpable warmth of the synovium. Additional inclusion criteria comprised a radiographic confirmed Kellgren and Lawrence grade II or III (mild to moderate osteophytes and joint space narrowing) in the previous 6 months [19]; a knee OA pain intensity score, assessed by the question "How would you describe your maximum OA knee pain when not taking analgesic medications in the $24 \mathrm{~h}$ prior to this visit", of "moderate (2)" or "moderately severe (3)" on a 5-point Likert Scale; a Western Ontario and McMaster University OA Index (WOMAC) physical function subscale score $>0$ on a $100 \mathrm{~mm}$ horizontal visual analogue scale (VAS) [20]. In case both knees were affected, the knee with the highest pain score was selected as the target knee. In order to avoid interference with the subject's pain perception, the use of paracetamol within a $48 \mathrm{~h}$ period prior to the screening visit was not allowed.

The major exclusion criteria were the following: secondary OA of the target knee; morning stiffness of $\geq 30 \mathrm{~min}$; a swollen or warm joint suspected to be secondary to gout, pseudo gout or sepsis; significant injury in the target joint within 6 months before the start of the trial; arthroplasty and joint surgery of the target knee within 2 years prior to the start of the study. Additionally, specified previous medications led to exclusion from the trial.

Following a wash-out period during which the use of pain medication and OA-related treatments were not allowed, eligible subjects were scheduled for a baseline visit. The duration of the wash-out period was at least five drug half-lives of the corresponding drug, or as described in the exclusion criteria. 


\section{Design, randomisation and blinding}

At the baseline visit, patients were randomly assigned to take a capsule of either ch-OSA (520 mg ch-OSA beadlets containing $5 \mathrm{mg}$ of silicon and $100 \mathrm{mg}$ of choline; Bio Minerals N.V., Belgium) or placebo (520 mg microcrystalline cellulose beadlets; Pharmatrans Sanaq AG, Switzerland) twice daily by oral route for 12 weeks. The treatment allocation occurred sequentially in a 1:1 ratio using a randomization list, which was generated by an independent statistician in R (software version 2.10.1 for Windows; The R Foundation for Statistical Computing, Vienna, Austria). More specifically, block randomization was stratified by site, applying randomly selected block sizes of 2 or 4 . The individual code was kept in a sealed envelope by the investigator to be opened only in case of medical emergency.

Patients were assessed by the investigator at baseline and at 2, 6 and 12 weeks after randomization. At the first three visits, the study dietary supplement was delivered in bottles labelled with the subject's randomization number (according to the allocation sequence) and the instructions to take one capsule in the morning and another in the evening with a glass of water or juice. Blinding among subjects, investigators and monitors was maintained by providing identical packaging, appearance, taste and odour for ch-OSA and placebo capsules, respectively. Treatment compliance was verified at subsequent visits by counting the number of unused doses. In this respect, the limit for an acceptable compliance was set at $75 \%$.

Pain medication and OA-related treatments were not allowed throughout the trial. However, subjects were allowed to take up to $2 \mathrm{~g}$ of paracetamol per day as rescue medication in case of intolerable pain, except during a $48 \mathrm{~h}$ period before each evaluation.

\section{Assessments and outcome measures}

At each visit, allocated patients were requested to complete a $100 \mathrm{~mm}$ horizontal VAS WOMAC questionnaire based on symptoms in the target knee in the preceding $48 \mathrm{~h}$. This 24-item questionnaire resulted in a total WOMAC score and three WOMAC subscores, based on the following subscales: pain (5 questions), stiffness (2 questions) and physical function (17 questions). For each WOMAC score, a 0$100 \mathrm{~mm}$ range was generated by averaging across the respective items, with lower scores indicating better outcomes. The Subject Global Assessment was evaluated on a $100 \mathrm{~mm}$ horizontal VAS as well $(0-100 \mathrm{~mm}$ range), by asking the following question: "Considering all the ways your knee OA affects you, how are you doing today?". Finally, fasting serum and second void urine were collected at the baseline and final visit to assess biochemical markers of cartilage degradation (urine, serum), baseline serum estradiol and serum silicon concentration. For the collection of serum, silicon free polypropylene syringes (Sarstedt, Germany) and needles (Microlance, Becton Dickinson,
Spain) were used as published elsewhere [15]. Aliquots of both samples were stored at $-20{ }^{\circ} \mathrm{C}$ until analysis. More specifically, urinary CTX-II concentrations were determined using the Urine CartiLaps EIA from IDS (Boldon, UK). These concentrations were corrected for urinary creatinine concentrations, which were determined with a creatinine assay (R\&D Systems, Abingdon, UK). Serum COMP levels were measured using AnaMar Medical's (Lund, Sweden) COMP ELISA. Serum Estradiol levels were measured with Estradiol III electrochemiluminescense immunoassay from Roche (Mannheim, Germany). All analyses were performed according to the manufacturers' protocols. Serum silicon concentration was measured using electrothermal atomic absorption spectrometry with inverse longitudinal Zeeman background correction (AAnalyst 800, Perkin Elmer, Bodenseewerk, Germany) and the analytical features (temperature program, matrix modifier, sample preparation method) were published elsewhere [15].

The primary outcome measure was the change in the WOMAC pain subscale from baseline to week 12 . Secondary outcome measures included the changes from baseline to 12 weeks in the total WOMAC score, the WOMAC stiffness subscale, the WOMAC physical function subscale, the Subject Global Assessment and CTX-II and COMP levels.

\section{Statistical analysis}

The sample size calculation estimated that a group size of 100 subjects was necessary to ensure at least $80 \%$ power to detect differences of $16 \%$ between the ch-OSA and placebo group with respect to pain and physical function (quantified according to the WOMAC pain and function subscale, assuming a relative standard deviation of $40 \%$ (based on data of 13 studies; Additional file 2: Table S2)), considering a dropout rate of $10 \%$ and a 0.05 two-sided significance level.

Statistical analyses were performed using the perprotocol population, defined as all randomized patients meeting the inclusion criteria, who completed the trial and who did not have major protocol violations. Results are presented as mean \pm standard deviation (SD).

For WOMAC total, WOMAC subscales and Subject Global Assessment scores, a repeated measures (RM) univariate analysis of covariance (ANCOVA) model was applied in which the baseline value was treated as a covariate while treatment and gender were treated as fixed factors. Between and within treatment differences were assessed and the models also included interaction terms between treatment and gender. Differences in serum silicon and biomarker values (baseline versus final visit) were analysed between and within treatments using RM analysis of variance (ANOVA), albeit after removal of outliers. These outliers were identified using the median absolute deviation and a critical value of 4.5 [21]. In case of a significant interaction between time and treatment, post hoc t-tests with Bonferroni correction were performed. 
Additionally, as specified in the study protocol, subgroup analyses by gender were performed for all outcome measures. Due to the smaller group sizes, non-parametric tests were used. More specifically, differences between treatment groups were analysed with Mann-Whitney U, whereas differences within treatment groups were analysed using Friedman's test with post hoc Bonferronicorrected Wilcoxon signed-ranks. Correlation of serum silicon with clinical parameters was analysed in subgroups with the Spearman rank correlation test.

Significance levels were set at $P<0.05$ (two-sided), except for interaction tests $(P<0.10)$ [22]. All statistical analyses were performed using SPSS software (version 21.0 for Windows, IBM Corp., Armonk, NY, USA).

\section{Results}

\section{Patients}

Between June 2010 and April 2012, a total of 211 eligible subjects were randomized and allocated to receive
ch-OSA $(n=106)$ or a placebo $(n=105)$. As demonstrated in Fig. 1, 166 patients were ultimately included in the per-protocol analysis. Demographic variables, baseline disease characteristics and baseline outcome measures were similar in both groups (Table 1). Mean age of the randomized patients was $61.9 \pm 7.1$ years with a majority of postmenopausal women. The mean baseline serum level of estradiol was significantly $(P<0.0005)$ lower in women compared to men (Table 1). Baseline CTX-II levels showed a higher trend $(P=0.1)$ in women compared to men (Table 1).

\section{Compliance and safety}

Compliance throughout the study was excellent as $98 \%$ of the patients reached the minimum compliance of $75 \%$. Overall, the mean compliance was $106 \pm 12 \%$ in the per-protocol population and $>100 \%$ both in men and women. No adverse events related to the treatment were reported in either treatment group.

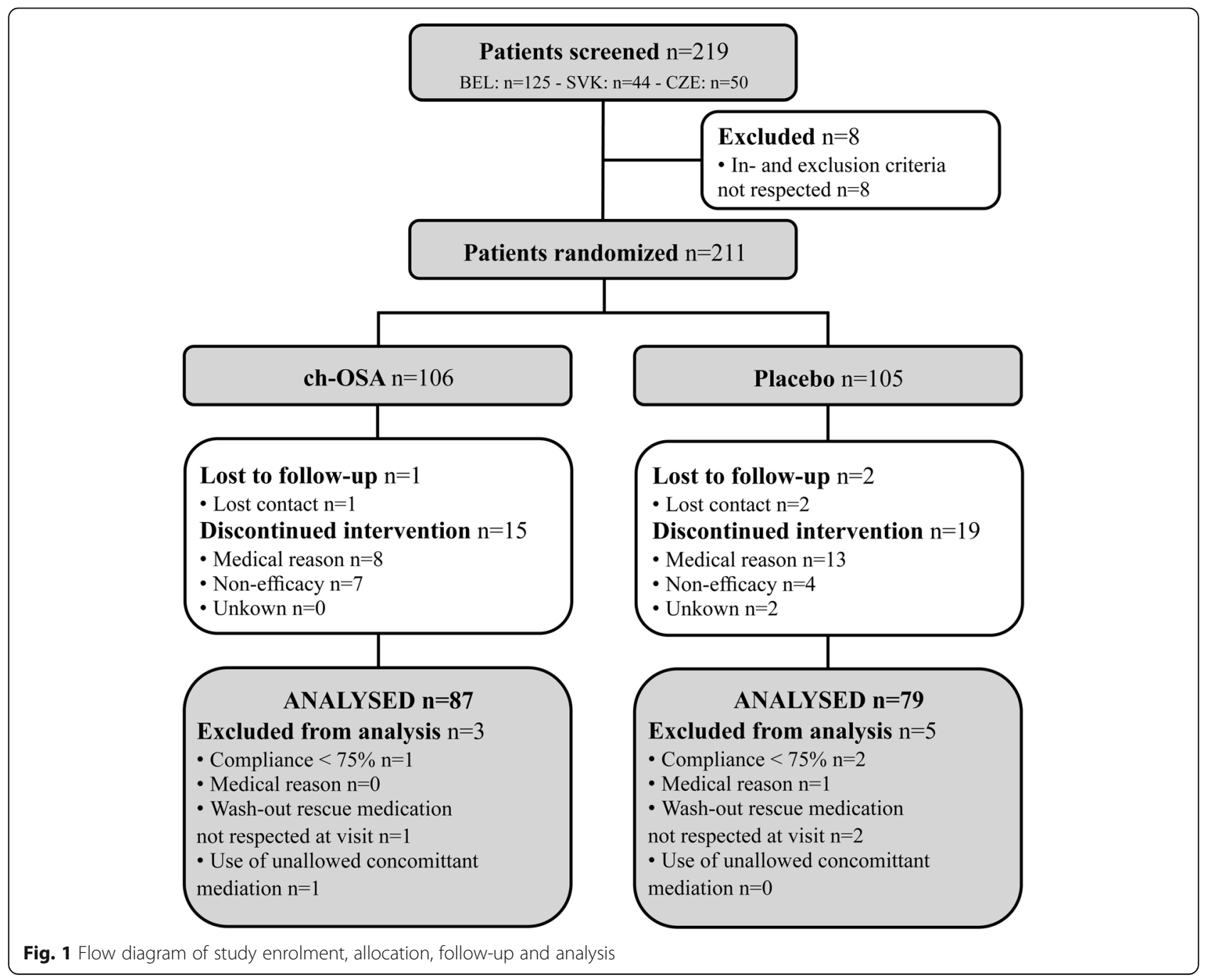


Table 1 Baseline characteristics of the study population by treatment group and gender

\begin{tabular}{|c|c|c|c|c|}
\hline Characteristic & Placebo $(n=79)$ & ch-OSA $(n=87)$ & Male $(n=46)$ & Female $(n=120)$ \\
\hline Age (years) & $62.2 \pm 7.7$ & $61.7 \pm 6.5$ & $61.0 \pm 7.2$ & $62.3 \pm 7.0$ \\
\hline Women & $55(69.6)$ & $65(74.7)$ & 0 & $120(100)$ \\
\hline Body mass index $\left(\mathrm{kg} / \mathrm{m}^{2}\right)$ & $29.5 \pm 5.2$ & $29.5 \pm 5.4$ & $29.1 \pm 4.7$ & $29.6 \pm 5.6$ \\
\hline \multirow[t]{3}{*}{ Menopausal Status } & 1 pre (1.5) & 1 pre (1.8) & - & 2 pre (1.7) \\
\hline & 0 peri (1.5) & 1 peri & & 1 peri (0.8) \\
\hline & 54 post (98.2) & 63 post (96.9) & & 117 post (97.5) \\
\hline Kellgren and Lawrence grade II & $48(60.8)$ & $59(67.8)$ & $26(56.5)$ & $81(67.5)$ \\
\hline Kellgren and Lawrence grade III & $31(39.2)$ & $28(32.2)$ & $20(43.5)$ & $39(32.5)$ \\
\hline Likert pain score 2 & $38(48.1)$ & $32(36.8)$ & $19(41.3)$ & $51(42.5)$ \\
\hline Likert pain score 3 & $41(51.9)$ & $55(63.2)$ & $27(58.7)$ & $69(57.5)$ \\
\hline WOMAC Total (/100 mm) & $43.1 \pm 20.3$ & $40.9 \pm 19.4$ & $42.2 \pm 21.5$ & $41.9 \pm 19.2$ \\
\hline WOMAC Pain (/100 mm) & $41.2 \pm 20.3$ & $38.2 \pm 19.8$ & $41.9 \pm 20.9$ & $38.7 \pm 19.7$ \\
\hline WOMAC Stiffness (/100 mm) & $45.3 \pm 24.6$ & $44.0 \pm 23.5$ & $44.9 \pm 23.4$ & $44.5 \pm 24.3$ \\
\hline WOMAC Function (/100 mm) & $43.4 \pm 21.2$ & $41.4 \pm 19.8$ & $42.0 \pm 22.5$ & $42.5 \pm 19.7$ \\
\hline Subject Global Assessment (/100 mm) & $49.4 \pm 21.8$ & $50.1 \pm 18.2$ & $51.3 \pm 22.4$ & $49.2 \pm 19.0$ \\
\hline CTX-II/Creatinine (ng/mmol) & $413.1 \pm 189.6$ & $430.4 \pm 212.7$ & $378.5 \pm 162.8$ & $441.0 \pm 213.9$ \\
\hline $\operatorname{COMP}(\mathrm{U} / \mathrm{L})$ & $10.8 \pm 2.8$ & $10.7 \pm 2.8$ & $11.0 \pm 2.8$ & $10.6 \pm 2.8$ \\
\hline Estradiol (ng/L) & - & - & $35.5 \pm 31.0$ & $22.0 \pm 36.2^{a}$ \\
\hline
\end{tabular}

Data expressed as mean \pm SD or $n(\%) ;-:$ not applicable; ${ }^{a}$ significant difference between groups (men versus women); Number of patients without outliers for CTX-II: Placebo $(n=66)$, ch-OSA $(n=68)$; Number of patients without outliers for COMP: Placebo $(n=74)$, ch-OSA $(n=80)$

\section{Efficacy}

The primary and secondary outcome measures are shown in Table 2, along with the absolute values of the assessed clinical outcome measures at the different visits. After 12 weeks of treatment, the change from baseline in silicon serum levels was significantly higher in the chOSA group compared to the placebo group (Table 2). The statistical analyses demonstrated no significant differences between the two treatment groups with respect to the primary and secondary outcome measures. Nevertheless, significant treatment $\mathrm{x}$ gender interactions were found for all clinical outcomes (ANCOVA).

Subsequently, pre-specified subgroup analyses by gender were performed. Table 3 provides an overview of the assessed parameters in men and women in the placebo and ch-OSA group, respectively. There was a superior effect of ch-OSA in men after 12 weeks of treatment as the mean changes from baseline in WOMAC total, WOMAC stiffness and WOMAC physical function were significantly higher with ch-OSA compared to placebo (Fig. 2). The corresponding mean differences $(95 \% \mathrm{CI})$ between placebo and ch-OSA in men were the following: WOMAC total $11.0 \mathrm{~mm}$ (0.1 to 21.9), WOMAC stiffness $16.5 \mathrm{~mm}$ (3.0 to 30.0) and WOMAC physical function $10.6 \mathrm{~mm}$ ( -0.5 to 21.6). A similar, yet non-significant trend was observed for WOMAC pain and Subject Global Assessment: $10.2 \mathrm{~mm}$ $(-1.7$ to 22.1$)$ and $8.4 \mathrm{~mm}$ (-5.5 to 22.3$)$, respectively. As shown in Fig. 3, increases in biomarkers of cartilage degradation (CTX-II and COMP) were significantly lower after 12 weeks in the male ch-OSA group, resulting in the following mean differences (95\% CI) between placebo and ch-OSA: CTX-II $102.0 \mathrm{ng} / \mathrm{mmol}$ (12.6 to 191.4) (Fig. 3a) and COMP $2.1 \mathrm{U} / \mathrm{L}$ (0.3 to 3.9) (Fig. 3b). The statistical analysis of the female subgroups, on the other hand, demonstrated no significant treatment differences (data not shown). Similar as in the total study population, the change from baseline in silicon serum levels was significantly higher in the ch-OSA group compared to the placebo group for both women and men. The following observed mean differences $(95 \% \mathrm{CI})$ between placebo and ch-OSA were observed: men, $26.1 \mu \mathrm{g} / \mathrm{L}$ (3.15 to 49.4) and women, $46.0 \mu \mathrm{g} / \mathrm{L}$ (27.0 to 65.0). No significant correlations were found between clinical parameters and serum silicon in neither women nor men (data not shown).

\section{Discussion}

This is the first clinical study to evaluate the symptom relief effect of ch-OSA in patients with knee OA. Although the intake of the dietary supplement for 12 weeks failed to show a statistically significant benefit over placebo regarding the clinical outcome scores and biomarker values in the per-protocol population, pre-specified subgroup analyses demonstrated a significant effect of ch-OSA in men. Accordingly, WOMAC total, stiffness and physical function scores were significantly reduced in men taking ch-OSA compared to men in the placebo group. While 


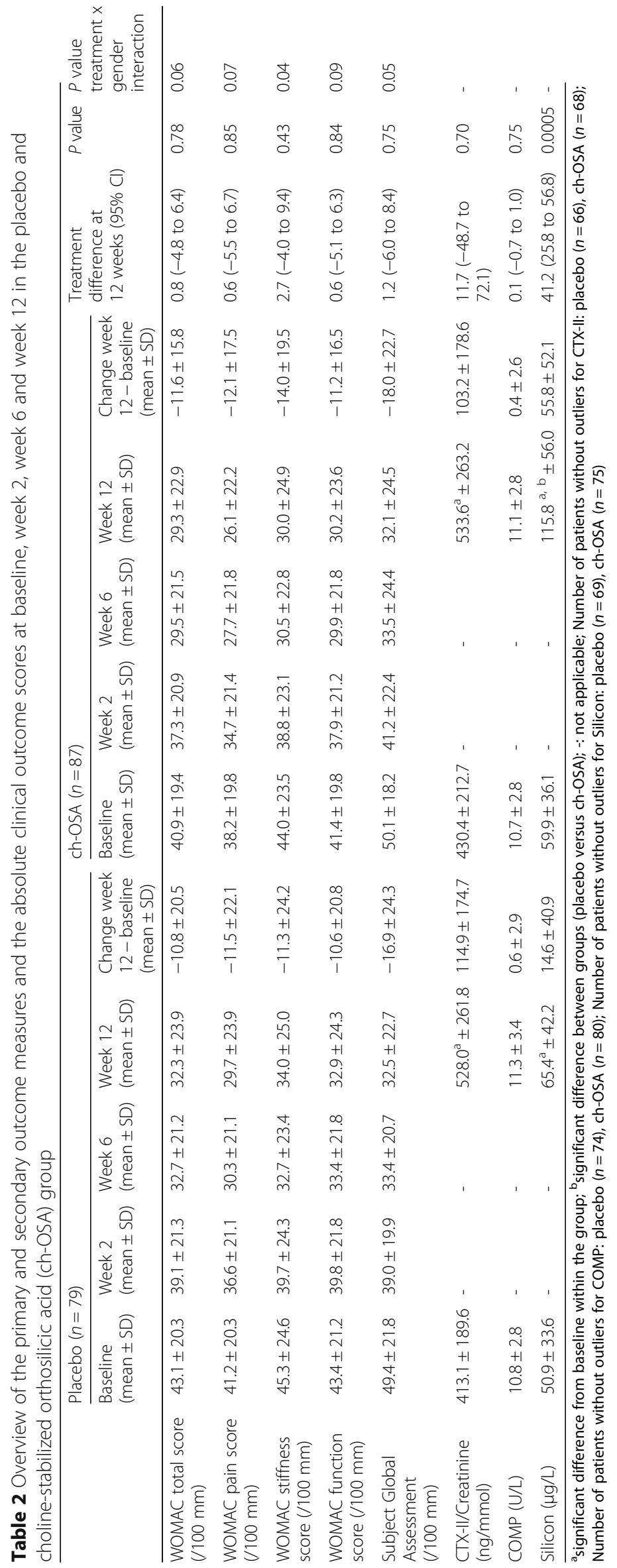


levels of cartilage degradation related biomarkers increased over 12 weeks in men in the placebo group and in women in both groups, these levels were significantly lower in the male ch-OSA group at the final visit. More specifically, the increase in CTX-II was less pronounced and COMP levels remained unchanged. The former suggests that ch-OSA can slow the ongoing progression of cartilage degradation in men.

The management of knee OA remains a major challenge. The key objective is to cease or delay disease progression by controlling cartilage degradation, inflammation and changes of subchondral bone. Nevertheless, most published recommendations primarily aim to control OA symptoms since safe and effective disease-modifying OA drugs (DMOADs) are currently lacking [23-26]. NSAIDs, for instance, are still among the most commonly used medicines to relieve OA pain, regardless of their well-known sideeffects in prolonged therapy $[24,27]$. The rationale for studying the effectiveness of ch-OSA in knee OA was consequently based on its previously demonstrated effect on collagen synthesis and its safety in long-term use [14, 15]. The components of ch-OSA, choline and orthosilicic acid (OSA), have both been mentioned with respect to collagen metabolism. Firstly, choline lowers plasma homocysteine levels through its precursor-function in the biochemical conversion of homocysteine to methionine [16]. This reduction positively affects collagen cross-linking, since homocysteine has been shown to interfere with posttranslational modifications of collagen through direct and indirect inhibition of lysyl oxidase as well as through down regulation of other genes involved in collagen cross-linking
[28]. Indeed, Zhang et al. recently reported elevated serum homocysteine levels in patients with severe OA compared to those with mild OA or healthy controls [29]. Secondly, although the exact role of silicon in bone and connective tissue health remains unclear, its involvement in collagen synthesis and/or stabilisation was recently put forward by Jugdaohsingh et al. [30]. In the present study, the lack of correlation between the change in serum silicon and clinical parameters in men taking ch-OSA, suggests that the effect of ch-OSA cannot be explained as an effect of silicon only. ch-OSA was previously shown to have a positive effect on markers of bone formation, with a significant increase of procollagen type I N-terminal propeptide (PINP) in osteopenic women following a supplementation period of 12 months [14]. In an animal model for postmenopausal osteoporosis, it was demonstrated that ch-OSA partially prevented femoral bone loss [31].

The former supports the hypothesis of a possible effect of ch-OSA on collagen in both cartilage and subchondral bone and consequently on knee OA, particularly in view of increasing evidence of a close interrelationship between subchondral bone and articular cartilage [32, 33]. A positive effect of ch-OSA was indeed confirmed in the present study, already after 12 weeks of treatment, yet only in men. Several hypotheses can be suggested with respect to this gender difference. In general, women report more pain and disability from knee OA $[34,35]$. The perception of pain, however, has been demonstrated to depend on the estrogen state. In fact, a low estrogen state was found to be related to hyperalgesia [36]. Consequently, the possibility that the enhanced pain

Table 3 Gender-specific subgroups: Overview of the absolute clinical outcome scores and biomarker values at baseline, week 2, week 6 and week 12 in the placebo and choline-stabilized orthosilicic acid (ch-OSA) group

\begin{tabular}{|c|c|c|c|c|c|c|c|c|}
\hline & \multicolumn{8}{|c|}{ Placebo $(n=79)$} \\
\hline & \multicolumn{4}{|l|}{ Male $(n=24)$} & \multicolumn{4}{|c|}{ Female $(n=55)$} \\
\hline & $\begin{array}{l}\text { Baseline } \\
(\text { mean } \pm \text { SD) }\end{array}$ & $\begin{array}{l}\text { Week } 2 \\
(\text { mean } \pm \text { SD) }\end{array}$ & $\begin{array}{l}\text { Week } 6 \\
(\text { mean } \pm \text { SD) }\end{array}$ & $\begin{array}{l}\text { Week } 12 \\
(\text { mean } \pm \text { SD) }\end{array}$ & $\begin{array}{l}\text { Baseline } \\
(\text { mean } \pm \text { SD) }\end{array}$ & $\begin{array}{l}\text { Week } 2 \\
(\text { mean } \pm \text { SD) }\end{array}$ & $\begin{array}{l}\text { Week } 6 \\
(\text { mean } \pm \text { SD) }\end{array}$ & $\begin{array}{l}\text { Week } 12 \\
(\text { mean } \pm \mathrm{SD})\end{array}$ \\
\hline $\begin{array}{l}\text { WOMAC total score } \\
(/ 100 \mathrm{~mm})\end{array}$ & $42.0 \pm 22.4$ & $37.5^{a} \pm 26.2$ & $34.1^{\mathrm{a}} \pm 24.3$ & $34.7 \pm 30.7$ & $43.6 \pm 19.6$ & $39.8 \pm 19.0$ & $32.1^{a, b} \pm 19.9$ & $31.2^{a} \pm 20.5$ \\
\hline $\begin{array}{l}\text { WOMAC pain score } \\
(/ 100 \mathrm{~mm})\end{array}$ & $42.5 \pm 22.7$ & $35.1^{a} \pm 24.5$ & $32.6^{a} \pm 24.0$ & $33.0 \pm 31.2$ & $40.6 \pm 19.4$ & $37.2 \pm 19.7$ & $29.3^{\mathrm{a}, \mathrm{b}} \pm 19.8$ & $28.2^{a} \pm 20.1$ \\
\hline $\begin{array}{l}\text { WOMAC stiffness score } \\
(/ 100 \mathrm{~mm})\end{array}$ & $43.8 \pm 25.3$ & $40.0 \pm 27.3$ & $33.5 \pm 26.4$ & $38.2 \pm 29.6$ & $45.9 \pm 24.5$ & $39.5^{\mathrm{a}} \pm 23.2$ & $32.4^{\mathrm{a}, \mathrm{b}} \pm 22.2$ & $32.2^{a} \pm 22.8$ \\
\hline $\begin{array}{l}\text { WOMAC function score } \\
(/ 100 \mathrm{~mm})\end{array}$ & $41.7 \pm 23.3$ & $37.9 \pm 27.3$ & $34.6 \pm 25.2$ & $34.9 \pm 31.0$ & $45.9 \pm 24.5$ & $39.5 \pm 23.2$ & $32.4^{a, b} \pm 22.2$ & $32.2^{a} \pm 22.8$ \\
\hline $\begin{array}{l}\text { Subject Global Assessment } \\
(/ 100 \mathrm{~mm})\end{array}$ & $51.1 \pm 26.0$ & $41.7 \pm 22.4$ & $33.3^{a} \pm 23.4$ & $33.8^{\mathrm{a}} \pm 24.3$ & $48.6 \pm 19.9$ & $37.9^{a} \pm 18.8$ & $33.5^{a, b} \pm 19.7$ & $32.0^{a} \pm 22.2$ \\
\hline CTX-II/Creatinine (ng/mmol) & $389.8 \pm 178.0$ & - & - & $565.2^{a} \pm 267.8$ & $425.6 \pm 196.5$ & - & - & $508.1^{a} \pm 259.5$ \\
\hline $\operatorname{COMP}(\mathrm{U} / \mathrm{L})$ & $11.1 \pm 3.0$ & - & - & $13.0^{\mathrm{a}} \pm 3.6$ & $10.6 \pm 2.7$ & - & - & $10.6 \pm 3.2$ \\
\hline Silicon ( $\mu \mathrm{g} / \mathrm{L})$ & $54.4 \pm 28.1$ & - & - & $59.2 \pm 36.0$ & $49.5 \pm 35.6$ & & & $67.8^{a} \pm 44.4$ \\
\hline
\end{tabular}

${ }^{a}$ significant difference from baseline within the group; ${ }^{b}$ significant difference from week 2 within the group; ${ }^{c}$ significant difference between groups (placebo versus ch-OSA); -: not applicable; Number of patients without outliers for CTX-Il: placebo males $(n=23)$, placebo females $(n=43)$, ch-OSA males $(n=18)$, ch-OSA females $(n=50)$; Number of patients without outliers for COMP: placebo males $(n=22)$, placebo females $(n=52)$, ch-OSA males $(n=21)$, ch-OSA females $(n=59)$; Number of patients without outliers for Silicon: placebo males $(n=19)$, placebo females $(n=50)$, ch-OSA males $(n=19)$, ch-OSA females $(n=56)$ 
Table 3 Gender-specific subgroups: Overview of the absolute clinical outcome scores and biomarker values at baseline, week 2, week 6 and week 12 in the placebo and choline-stabilized orthosilicic acid (ch-OSA) group (Continued)

\begin{tabular}{|c|c|c|c|c|c|c|c|c|}
\hline & \multicolumn{8}{|c|}{ ch-OSA $(n=87)$} \\
\hline & \multicolumn{4}{|l|}{ Male $(n=22)$} & \multicolumn{4}{|c|}{ Female $(n=65)$} \\
\hline & $\begin{array}{l}\text { Baseline } \\
(\text { mean } \pm \text { SD) }\end{array}$ & $\begin{array}{l}\text { Week } 2 \\
(\text { mean } \pm \mathrm{SD})\end{array}$ & $\begin{array}{l}\text { Week } 6 \\
(\text { mean } \pm S D)\end{array}$ & $\begin{array}{l}\text { Week } 12 \\
(\text { mean } \pm \text { SD) }\end{array}$ & $\begin{array}{l}\text { Baseline } \\
\text { (mean } \pm \text { SD) }\end{array}$ & $\begin{array}{l}\text { Week } 2 \\
(\text { mean } \pm \text { SD) }\end{array}$ & $\begin{array}{l}\text { Week } 6 \\
(\text { mean } \pm \text { SD) }\end{array}$ & $\begin{array}{l}\text { Week } 12 \\
(\text { mean } \pm \text { SD) }\end{array}$ \\
\hline $\begin{array}{l}\text { WOMAC total score } \\
(/ 100 \mathrm{~mm})\end{array}$ & $42.5 \pm 20.9$ & $36.1^{a} \pm 22.1$ & $26.8^{a} \pm 22.8$ & $24.2^{a} \pm 21.3$ & $40.4 \pm 19.0$ & $37.7 \pm 20.7$ & $30.4^{a, b} \pm 21.2$ & $31.1^{\mathrm{a}} \pm 23.4$ \\
\hline $\begin{array}{l}\text { WOMAC pain score } \\
(/ 100 \mathrm{~mm})\end{array}$ & $41.3 \pm 19.3$ & $32.2^{a} \pm 20.0$ & $24.3^{a} \pm 21.5$ & $21.6^{a} \pm 19.6$ & $37.1 \pm 20.0$ & $35.5 \pm 21.9$ & $28.8^{\mathrm{a}, \mathrm{b}} \pm 22.0$ & $27.6^{\mathrm{a}} \pm 22.9$ \\
\hline $\begin{array}{l}\text { WOMAC stiffness score } \\
(/ 100 \mathrm{~mm})\end{array}$ & $46.2 \pm 21.8$ & $37.2^{\mathrm{a}} \pm 25.3$ & $28.8^{a} \pm 23.8$ & $24.1^{\mathrm{a}} \pm 23.4$ & $43.3 \pm 24.1$ & $39.4 \pm 22.5$ & $31.1^{\mathrm{a}, \mathrm{b}} \pm 22.6$ & $32.0^{\mathrm{a}} \pm 25.3$ \\
\hline $\begin{array}{l}\text { WOMAC function score } \\
(/ 100 \mathrm{~mm})\end{array}$ & $42.4 \pm 22.2$ & $27.1 \pm 22.9$ & $27.3^{\mathrm{a}} \pm 23.5$ & $25.0^{\mathrm{a}} \pm 22.3$ & $43.3 \pm 24.1$ & $39.4 \pm 22.5$ & $31.1^{\mathrm{a}, \mathrm{b}} \pm 22.6$ & $32.0^{\mathrm{a}} \pm 25.3$ \\
\hline $\begin{array}{l}\text { Subject Global Assessment } \\
(/ 100 \mathrm{~mm})\end{array}$ & $51.5 \pm 18.3$ & $36.6^{a} \pm 20.9$ & $24.6^{a, b} \pm 20.8$ & $25.8^{\mathrm{a}} \pm 23.7$ & $49.6 \pm 18.3$ & $42.7^{\mathrm{a}} \pm 22.8$ & $36.5^{\mathrm{a}, \mathrm{b}} \pm 24.9$ & $34.2^{\mathrm{a}} \pm 24.6$ \\
\hline CTX-II/Creatinine (ng/mmol) & $364.0 \pm 144.7$ & - & - & $437.4^{\mathrm{a}} \pm 171.8$ & $454.3 \pm 229.0$ & - & - & $568.3^{\mathrm{a}} \pm 282.6$ \\
\hline $\operatorname{COMP}(\mathrm{U} / \mathrm{L})$ & $10.9 \pm 2.7$ & - & - & $10.6 \pm 2.7$ & $10.6 \pm 2.9$ & - & - & $11.3^{\mathrm{a}} \pm 2.8$ \\
\hline Silicon $(\mu \mathrm{g} / \mathrm{L})$ & $68.4 \pm 38.0$ & - & - & $99.3^{a, c} \pm 42.7$ & $57.0 \pm 35.3$ & - & - & $121.3^{a, c} \pm 59.1$ \\
\hline
\end{tabular}

perception masked the effect of ch-OSA in women in the present study cannot be ruled out given the predominance of postmenopausal women (98\%) with low endogenous estrogen levels, of which only $7 \%$ was on hormone replacement therapy (HRT), a contraceptive, a selective estrogen receptor modulator (SERM) or phytoestrogens. In fact, the mean serum estradiol level of women in the present study was significantly lower compared to that of the male study population.

Besides the fact that postmenopausal women have a different pain perception than man, it has also been confirmed that the severity of knee OA is higher in women as well, and principally in postmenopausal women $[9,37]$. Accordingly, urinary CTX-II levels were found to be significantly higher in postmenopausal women than in men [38]. The latter is in correspondence with the trend towards higher baseline CTX-II concentrations observed in - predominantly postmenopausal - women in the present study. Again, decreased estrogen levels might be of importance. Several in vitro, in vivo, genetic and clinical studies indeed designate a potential protective role for estrogens against the development of $\mathrm{OA}$, given the various actions of estrogen on articular tissues [39]. Additionally, considerable experimental models indicate that estrogen deficiency-related increases in bone turnover contribute to the progression of OA [40, 41]. Therefore, in view of the more severe OA forms in women, it can be suggested that the 12-week supplementation period could have been insufficient to result in a clinical benefit.

Given the apparent extensive involvement of estrogen deficiency in the development and progression of knee OA, the use of HRTs or SERMs in women seems plausible. Nevertheless, it has been concluded that the health risks of HRTs may outweigh the potential benefits and that HRTs

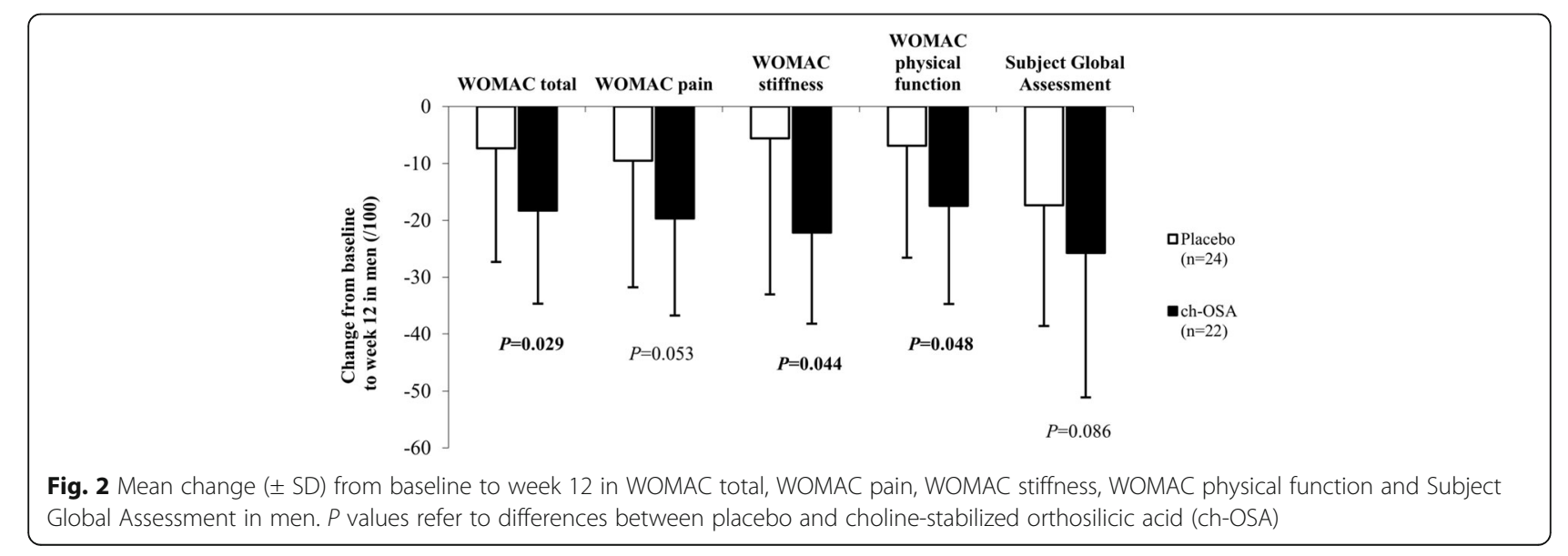



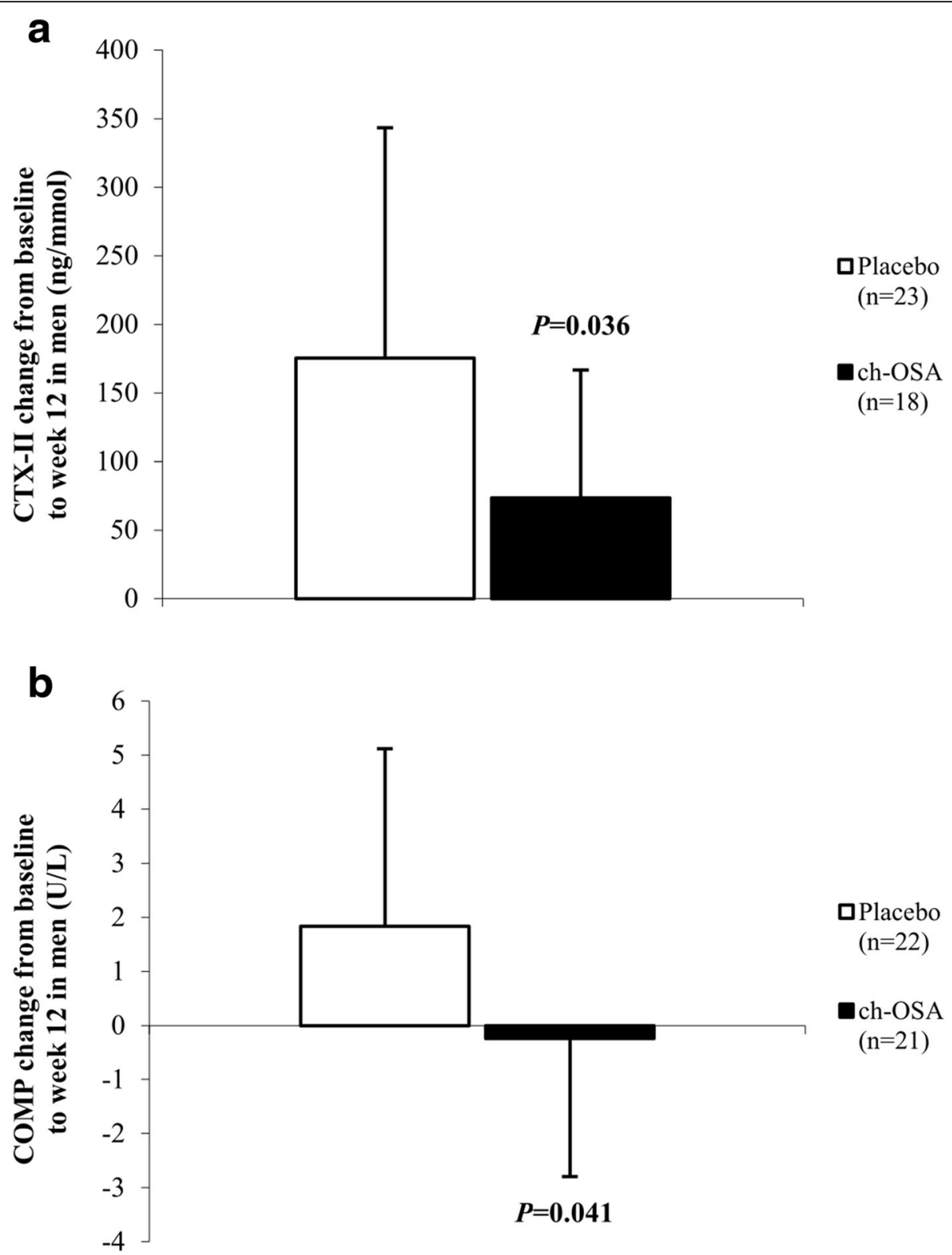

Fig. 3 Mean change ( \pm SD) from baseline to week 12 in CTX-II (a) and COMP (b) levels in men. $P$ values refer to differences between placebo and choline-stabilized orthosilicic acid (ch-OSA)

and SERMs should not be recommended as first-line treatments for OA [42, 43]. Several other pharmacological therapies have been studied with respect to knee OA, including symptomatic slow-acting drugs for OA (SYSADOAs) like glucosamine sulphate, chondroitin sulphate, hyaluronic acid and diacerein. Due to the frequently inconsistent conclusions of these studies, the appropriateness of the former therapies has been recently designated as uncertain [26]. Furthermore, therapeutic approaches that target subchondral bone resorption and/or formation have gained attention [32, 43]. Bisphosphonates, strontium ranelate and calcitonin have been studied in this context, yet none of these treatments has been approved as a DMOAD since evidence of concomitant structural and symptomatic effects is still pending $[6,26]$. Additionally, safety issues are of importance, such as the risk of cardiovascular events associated with strontium ranelate [44]. The safety of ch-OSA in prolonged therapy, on the other hand, has been previously demonstrated [45].

Although the relevance of biochemical markers in OA has not yet been proven to be of definite clinical importance, Lotz et al. described their value as secondary outcomes [12]. More specifically, effects on biomarkers can support the primary outcome and provide evidence of 
pharmacodynamics and mechanisms of action of $\mathrm{OA}$ drugs. This is indeed valid in the present study, given that the trend observed for the primary outcome (WOMAC pain) in ch-OSA-treated men was associated with significantly lower biomarker levels, the latter referring to decreased cartilage degradation.

The present study has a number of limitations. Firstly, the evaluation of the magnitude of pain changes is ambiguous. This is related to the relatively high placebo effect - which is however common in OA trials - on the one hand, and to the complicated interpretation of changes evaluated as means rather than individual improvements, on the other hand [46]. Furthermore, the dropout rate was higher than assumed in the sample size calculation (18\% in contrast to the postulated $10 \%)$, which negatively affected the statistical power in the subgroup analyses, particularly in the male subgroups.

In conclusion, this randomized, placebo-controlled trial provided a first indication of a potential benefit of ch-OSA in the treatment of knee OA in men, particularly in view of the need for a safe long-term treatment. Future research is however needed to further elucidate the observed effects and gender difference since no benefit was found in women. Considering the hypothesis that the 12-week supplementation period may have been insufficient in women, an extension of the treatment duration to at least 6 months would be valuable [47]. Additionally, the inclusion of younger, premenopausal women could, at least in part, contribute to the clarification of the role of estrogen in OA. The potential disease-modifying effects of ch-OSA at the level of the knee, on the other hand, could be evaluated after a prolonged treatment period using reliable and sensitive imaging technologies, such as MRI or highresolution peripheral quantitative computed tomography (HRpQCT) [43, 48]. Determination of biochemical markers of bone metabolism could also extend the knowledge on the mechanisms of action of chOSA. Finally, application of the former research suggestions would also contribute to the identification of different subgroups. These subgroups could then be subjected to separate statistical analyses, which could provide evidence for the further assessment of ch-OSA's potentials in view of individualized targeted treatments for knee OA.

\section{Conclusions}

Choline-stabilized orthosilicic acid did not improve symptoms of knee osteoarthritis in the total study population but a gender effect was observed. A symptomatic improvement was found in men but not in women, after 12 weeks of supplementation, which was associated with a slight but significant reduction of biomarkers which are related to cartilage degradation. In view of the need for a safe long-term therapy for the treatment of knee osteoarthritis, this study provided first evidence on the potential benefit of choline-stabilized orthosilicic acid. Future research is however needed to further elucidate the observed effects and gender difference. Considering the hypothesis that the 12-week supplementation period may have been insufficient in women, an extension of the treatment duration to at least 6 months would be valuable.

\section{Additional files}

Additional file 1: Table S1. Inclusion and exclusion criteria. (DOC 29 kb)

Additional file 2: Table S2. References applies to determine the relative standard deviation (power calculation). (DOCX $13 \mathrm{~kb}$ )

Additional file 3: Table S3. Protocol violations resulting in exclusion from the per-protocol population. (DOC $24 \mathrm{~kb}$ )

Additional file 4: Dataset Geusens et al. knee OA study. (XLS 112 kb)

\section{Abbreviations}

ANCOVA: Univariate analysis of covariance; BIPED: Burden of disease, investigative, prognostic, efficacy of intervention and diagnostics; chOSA: choline-stabilized orthosilicic acid; COMP: Cartilage oligomeric matrix protein; CTX-II: C-terminal telopeptide of collagen type II; DMOADs: Diseasemodifying osteoarthritis drugs; ELISA: Enzyme-linked immuno sorbent assay; HRpQCT: High-resolution peripheral quantitative computed tomography; HRT: Hormone replacement therapy; MRI: Magnetic resonance imaging; NSAIDs: Non-steroidal anti-inflammatory drugs; OA: Osteoarthritis; OSA: Orthosilicic acid; PINP: Procollagen type I N-terminal propeptide; RM: Repeated measures; SERM: Selective estrogen receptor modulator; SYSADOAs: Symptomatic slow-acting drugs for osteoarthritis; VAS: Visual analogue scale; WOMAC: Western Ontario and McMaster University osteoarthritis Index

\section{Acknowledgements}

The authors thank Veronika Sebkova, Eva Kracmarova, Marianna Provazníková, Eva Jančušková, Adéla Suchá and Marcela Kadlecová from MKS Research s.r.o. for monitoring activities and trial coordination in Slovakia and the Czech Republic; and Atlantic Bone Screen for the analysis of cartilage degradation markers. The following investigators participated in the trial: (Belgium) Marleen Coppens, Hubert Berghs, Willy Pardon, Sonja Poesen, Pascal Duysters, Jan Huysmans, Theo Vansummeren; (Slovakia) Roman Fano, Marián Kovalančík, Pavol Masaryk, Zuzana Popracová, Jozef Zvarka, Roman Stančik; (Czech Republic) Hana Jarošová, Martina Votavová, Simona

Skácelová. The help of the study nurses was also appreciated by the authors: (Belgium) Luc Emonds, Carine Swinnen, Els Binnemans; (Slovakia) Gabriela Klementová; (Czech Republic) Kateřina Kohoutová.

\section{Funding}

Bio Minerals N.V. provided the study medication (ch-OSA and placebo) and funding.

Availability of data and materials

All of the data supporting the present findings are contained within the manuscript and Additional files 1, 2, 3 and 4

Authors' contributions

Concept and study design, data interpretation, drafting and revising of the manuscript, final approval of the manuscript, agreement to be accountable for accuracy and integrity of the work: PG, KP, JR, JVH, ND, MC, DVB.

\section{Competing interests}

Dr. Geusens provides lectures, consulting advice and collaboration in clinical studies with Amgen, Eli Lilly, Merck Sharp and Dohme, Novartis, Sanofi-Aventis, Abbvie, Will, Pfizer, UCB, Bristol-Myers-Squibb, and Menarini. 
Dr. Pavelka received honoraria for speaking and consultations from BMS, AbbVie, MSD, Roche, Amgen, UCB, Egis, Pfizer, IBSA, GBC.

Dr. Demeester and Dr. Calomme are employees of the study sponsor Bio Minerals N.V.

\section{Consent for publication}

Not applicable.

\section{Ethics approval and consent to participate}

Written informed consent was obtained from all patients prior to randomization. The Ethics Board of Hasselt University (Belgium) functioned as central Ethics Committee and granted approval for the trial on March 2 2010 (reference of the approval: CME 2009/298), in addition the local Ethics Committees in Slovakia (National Institute of Rheumatic Diseases, Pieštany) and the Czech Republic (Ethics Committee on Clinical Trial on Human Medicinal Products, Prague) also approved the study protocol. All study methods and procedures were conducted in accordance with the principles of the Declaration of Helsinki (1964) changed by the 29th World Medical Assembly at Tokyo (1975).

\section{Author details}

'Biomedical Research Institute (BIOMED), Hasselt University, Diepenbeek, Belgium. ${ }^{2}$ Rheumatology, Maastricht UMC, Maastricht, The Netherlands. ${ }^{3}$ ReumaClinic, Bretheistraat 149, Genk 3600, Belgium. ${ }^{4}$ Institute of Rheumatology, Prague, Czech Republic. ${ }^{5}$ Nábrežie I. Krasku 4782/4, Pieštany, Slovakia. ${ }^{6}$ Research \& Development, Bio Minerals NV, Destelbergen, Belgium. 'Department of Pharmaceutical Sciences, University of Antwerp, Antwerp-Wilrijk, Belgium.

Received: 27 May 2016 Accepted: 19 December 2016 Published online: 05 January 2017

\section{References}

1. Woolf AD, Pfleger B. Burden of major musculoskeletal conditions. Bull World Health Organ. 2003;81:646-56.

2. Zhang W, Moskowitz RW, Nuki G, Abramson S, Altman RD, Arden N, et al. OARSI recommendations for the management of hip and knee osteoarthritis, part I: critical appraisal of existing treatment guidelines and systematic review of current research evidence. OsteoarthrCartil. 2007:15:981-1000.

3. Kraus VB, Burnett B, Coindreau J, Cottrell S, Eyre D, Gendreau M, et al. Application of biomarkers in the development of drugs intended for the treatment of osteoarthritis. Osteoarthr Cartil. 2011:19:515-42.

4. Felson DT, Lawrence RC, Dieppe PA, Hirsch R, Helmick CG, Jordan JM, et al. Osteoarthritis: new insights. Part 1: the disease and its risk factors. Ann Intern Med. 2000;133:635-46.

5. Biilsma JW, Berenbaum F, Lafeber FP. Osteoarthritis: an update with relevance for clinical practice. Lancet. 2011;377:2115-26.

6. Lafeber FP, van Laar JM. Strontium ranelate: ready for clinical use as diseasemodifying osteoarthritis drug? Ann Rheum Dis. 2013;72:157-61.

7. Losina E, Weinstein AM, Reichmann WM, Burbine SA, Solomon DH, Daigle ME, et al. Lifetime risk and age at diagnosis of symptomatic knee osteoarthritis in the US. Arthritis Care Res. 2013;65:703-11.

8. Thysen S, Luyten FP, Lories RJ. Targets, models and challenges in osteoarthritis research. Dis Model Mech. 2015;8:17-30.

9. Srikanth VK, Fryer JL, Zhai G, Winzenberg TM, Hosmer D, Jones G. A metaanalysis of sex differences prevalence, incidence and severity of osteoarthritis. Osteoarthr Cartil. 2005:13:769-81.

10. Guermazi A, Hunter DJ, Roemer FW. Plain radiography and magnetic resonance imaging diagnostics in osteoarthritis: validated staging and scoring. J Bone Joint Surg Am. 2009;91 Suppl 1:54-62.

11. van Spil WE, DeGroot J, Lems WF, Oostveen JC, Lafeber FP. Serum and urinary biochemical markers for knee and hip-osteoarthritis: a systematic review applying the consensus BIPED criteria. Osteoarthr Cartil. 2010;18:605-12.

12. Lotz M, Martel-Pelletier J, Christiansen C, Brandi ML, Bruyere O, Chapurlat R, et al. Value of biomarkers in osteoarthritis: current status and perspectives. Ann Rheum Dis. 2013;72:1756-63.

13. Bauer DC, Hunter DJ, Abramson SB, Attur M, Corr M, Felson D, et al. Classification of osteoarthritis biomarkers: a proposed approach. Osteoarthr Cartil. 2006;14:723-7.
14. Spector TD, Calomme MR, Anderson SH, Clement G, Bevan L, Demeester N, et al. Choline-stabilized orthosilicic acid supplementation as an adjunct to calcium/vitamin D3 stimulates markers of bone formation in osteopenic females: a randomized, placebo-controlled trial. BMC Musculoskelet Disord. 2008;9:85.

15. Barel A, Calomme M, Timchenko A, De Paepe K, Demeester N, Rogiers V, et al. Effect of oral intake of choline-stabilized orthosilicic acid on skin, nails and hair in women with photodamaged skin. Arch Dermatol Res. 2005:297:147-53.

16. Ueland PM. Choline and betaine in health and disease. J Inherit Metab Dis. 2011:34:3-15.

17. Toohey Jl. Homocysteine toxicity in connective tissue: theories, old and new. Connect Tissue Res. 2008:49:57-61.

18. Altman R, Asch E, Bloch D, Bole G, Borenstein D, Brandt K, et al. Development of criteria for the classification and reporting of osteoarthritis. Classification of osteoarthritis of the knee. Diagnostic and Therapeutic Criteria Committee of the American Rheumatism Association. Arthritis Rheum. 1986:29:1039-49.

19. Kellgren JH, Lawrence JS. Radiological assessment of osteo-arthrosis. Ann Rheum Dis. 1957;16:494-502

20. Bellamy N, Buchanan WW, Goldsmith CH, Campbell J, Stitt LW. Validation study of WOMAC: a health status instrument for measuring clinically important patient relevant outcomes to antirheumatic drug therapy in patients with osteoarthritis of the hip or knee. J Rheumatol. 1988;15:1833-40.

21. Leys C, Ley C, Klein O, Bernard P, Licata L. Detecting outliers: do not use standard deviation around the mean, use absolute deviation around the median. J Exp Soc Psychol. 2013;49:764-6.

22. Ziegler D, Pritchett YL, Wang F, Desaiah D, Robinson MJ, Hall JA, et al. Impact of disease characteristics on the efficacy of duloxetine in diabetic peripheral neuropathic pain. Diabetes Care. 2007;30:664-9.

23. Jordan KM, Arden NK, Doherty M, Bannwarth B, Bijlsma JW, Dieppe P, et al. EULAR Recommendations 2003: an evidence based approach to the management of knee osteoarthritis: report of a Task Force of the Standing Committee for International Clinical Studies Including Therapeutic Trials (ESCISIT). Ann Rheum Dis. 2003:62:1145-55.

24. Zhang W, Moskowitz RW, Nuki G, Abramson S, Altman RD, Arden N, et al. OARSI recommendations for the management of hip and knee osteoarthritis, part II: OARSI evidence-based, expert consensus guidelines. Osteoarthr Cartil. 2008:16:137-62.

25. Hochberg MC, Altman RD, April KT, Benkhalti M, Guyatt G, McGowan J, et al. American College of Rheumatology 2012 recommendations for the use of nonpharmacologic and pharmacologic therapies in osteoarthritis of the hand, hip, and knee. Arthritis Care Res. 2012;64:465-74.

26. McAlindon TE, Bannuru RR, Sullivan MC, Arden NK, Berenbaum F, Bierma-Zeinstra SM, et al. OARSI guidelines for the non-surgical management of knee osteoarthritis. Osteoarthr Cartil. 2014;22:363-88.

27. Geusens P, Lems W. Efficacy and tolerability of lumiracoxib, a highly selective cyclo-oxygenase-2 (COX2) inhibitor, in the management of pain and osteoarthritis. Ther Clin Risk Manag. 2008;4:337-44.

28. Thaler R, Agsten M, Spitzer S, Paschalis EP, Karlic H, Klaushofer K, et al. Homocysteine suppresses the expression of the collagen cross-linker lysyl oxidase involving IL-6, Fli1, and epigenetic DNA methylation. J Biol Chem. 2011;286:5578-88.

29. Zhang Q, Li H, Zhang Z, Yang F, Chen J. Serum metabolites as potential biomarkers for diagnosis of knee osteoarthritis. Dis Markers. 2015;2015:684794

30. Jugdaohsingh $\mathrm{R}$, Watson Al, Pedro LD, Powell JJ. The decrease in silicon concentration of the connective tissues with age in rats is a marker of connective tissue turnover. Bone. 2015;75:40-8.

31. Calomme M, Geusens P, Demeester N, Behets GJ, D'Haese P, Sindambiwe JB, et al. Partial prevention of long-term femoral bone loss in aged ovariectomized rats supplemented with choline-stabilized orthosilicic acid. Calcif Tissue Int 2006;78:227-32.

32. Spector TD, Conaghan PG, Buckland-Wright JC, Garnero P, Cline GA, Beary JF, et al. Effect of risedronate on joint structure and symptoms of knee osteoarthritis: results of the BRISK randomized, controlled trial [ISRCTN01928173]. Arthritis Res Ther. 2005;7:R625-33.

33. Lories RJ, Luyten FP. The bone-cartilage unit in osteoarthritis. Nat Rev Rheumatol. 2011:7:43-9.

34. McAlindon TE, Snow S, Cooper C, Dieppe PA. Radiographic patterns of osteoarthritis of the knee joint in the community: the importance of the patellofemoral joint. Ann Rheum Dis. 1992;51:844-9. 
35. O'Connor MI, Hooten EG. Breakout session: gender disparities in knee osteoarthritis and TKA. Clin Orthop Relat Res. 2011;469:1883-5.

36. Smith YR, Stohler CS, Nichols TE, Bueller JA, Koeppe RA, Zubieta JK. Pronociceptive and antinociceptive effects of estradiol through endogenous opioid neurotransmission in women. J Neurosci. 2006;26:5777-85.

37. Hanna FS, Teichtahl AJ, Wluka AE, Wang Y, Urquhart DM, English DR, et al. Women have increased rates of cartilage loss and progression of cartilage defects at the knee than men: a gender study of adults without clinical knee osteoarthritis. Menopause. 2009;16:666-70.

38. van Spil WE, Drossaers-Bakker KW, Lafeber FP. Associations of CTX-II with biochemical markers of bone turnover raise questions on its tissue origin: data from CHECK, a cohort study of early osteoarthritis. Ann Rheum Dis. 2013;72:29-36.

39. Roman-Blas JA, Castaneda S, Largo R, Herrero-Beaumont G. Osteoarthritis associated with estrogen deficiency. Arthritis Res Ther. 2009:11:241.

40. Calvo E, Castaneda S, Largo R, Fernandez-Valle ME, Rodriguez-Salvanes F, Herrero-Beaumont G. Osteoporosis increases the severity of cartilage damage in an experimental model of osteoarthritis in rabbits. Osteoarthr Cartil. 2007;15:69-77.

41. Wang CJ, Huang CY, Hsu SL, Chen JH, Cheng JH. Extracorporeal shockwave therapy in osteoporotic osteoarthritis of the knee in rats: an experiment in animals. Arthritis Res Ther. 2014;16:R139.

42. Reginster JY, Kvasz A, Bruyere O, Henrotin Y. Is there any rationale for prescribing hormone replacement therapy (HRT) to prevent or to treat osteoarthritis? Osteoarthr Cartil. 2003;11:87-91.

43. Kwan Tat S, Lajeunesse D, Pelletier JP, Martel-Pelletier J. Targeting subchondral bone for treating osteoarthritis: what is the evidence? Best Pract Res Clin Rheumatol. 2010;24:51-70.

44. European Medicines Agency (EMA). Recommendation to restrict the use of Protelos/Osseor (strontium ranelate). EMA/258269/2013. 2013. URL: http:// www.ema.europa.eu/docs/en_GB/document_library/Press_release/2013/04/ WC500142507.pdf. Accessed 25 Apr 2013.

45. European Food Safety Authority (EFSA). Scientific opinion of the panel on food additives and nutrient sources added to food on choline-stabilised orthosilicic acid added for nutritional purposes to food supplements following a request from the European Commission. EFSA J. 2009;948:1-23.

46. Reginster JY, Badurski J, Bellamy N, Bensen W, Chapurlat R, Chevalier X, et al. Efficacy and safety of strontium ranelate in the treatment of knee osteoarthritis: results of a double-blind, randomised placebo-controlled trial. Ann Rheum Dis. 2013;72:179-86.

47. Herrero-Beaumont G, Ivorra JA, Del Carmen Trabado M, Blanco FJ, Benito P, Martin-Mola E, et al. Glucosamine sulfate in the treatment of knee osteoarthritis symptoms: a randomized, double-blind, placebo-controlled study using acetaminophen as a side comparator. Arthritis Rheum. 2007;56:555-67.

48. Geusens P, Chapurlat R, Schett G, Ghasem-Zadeh A, Seeman E, de Jong J, et al. High-resolution in vivo imaging of bone and joints: a window to microarchitecture. Nat Rev Rheumatol. 2014;10:304-13.

\section{Submit your next manuscript to BioMed Central and we will help you at every step:}

- We accept pre-submission inquiries

- Our selector tool helps you to find the most relevant journal

- We provide round the clock customer support

- Convenient online submission

- Thorough peer review

- Inclusion in PubMed and all major indexing services

- Maximum visibility for your research

Submit your manuscript at www.biomedcentral.com/submit

) Biomed Central 